15

\title{
Исследование теплового переноса в полупрозрачной среде
}

\author{
(C) Е.Ю. Шампаров
}

Институт фризических проблем им. П.Л. Капицы РАН,

119334 Москва, Россия

e-mail: shamparov@bk.ru

(Поступило в Редакцию 24 ноября 2016 г. В окончательной редакции 19 июня 2017 г.)

Получено аналитическое решение задачи по одномерному радиационно-кондуктивному переносу тепла в однородной изотропной серой среде вблизи к плоской диффузной непрозрачной поверхности и между двумя параллельными плоскостями с разной температурой. Проведены бесконвекционные измерения тепловых сопротивлений плоскопараллельных образцов вспененного полиэтилена в зависимости от числа слоев (толщины) без экранов и с экранами из тонкой алюминиевой фольги. Показана применимость приведенного теоретического подхода и представленной экспериментальной методики для измерения кондуктивной и лучистой теплопроводности легких теплозащитных материалов.

DOI: 10.21883/JTF.2018.01.45497.2109

При отсутствии конвективных потоков вещества свойства среды определяются двумя типами теплопереноса: теплопроводностью и тепловым излучением. В абсолютном большинстве случаев, за исключением исследований прозрачности атмосферы Земли [1], радиационный вклад в тепловой перенос полагают малым по сравнению с кондуктивным, что далеко не всегда справедливо. Собственно радиационно-кондуктивные задачи по передаче тепла чаще всего рассматривают в приложении к полупрозрачным материалам при высоких температурах $\left(\sim 1000^{\circ} \mathrm{C}\right)[2,3]$. В настоящей работе показано, что есть класс технически значимых легких теплозащитных материалов, в которых уже при обычных температурах радиационный и кондуктивный вклады сравнимы. Дан теоретический анализ свойств среды, построены методики и проведены непосредственные измерения радиационной и кондуктивной составляющих теплопроводности материала.

Рассмотрим радиационный поток тепла в полупрозрачной (серой) среде. Поток энергии или мощность, идущая через элемент площади $S$,

$$
P=(\Phi S)=\Phi S \cos \varphi,
$$

где $\Phi$ - плотность потока энергии.

Пусть у нас есть пространство, заполненное однородной изотропной средой с близким к 1 показателем преломления и независящей от частоты глубиной проникновения теплового излучения $a$. Проходя через слой некоторой толщины $d l$, излучение экспоненциально затухает

$$
\Phi=\Phi_{0} \exp (-d l / a)=\Phi_{0}(1-d l / a) .
$$

Поглощение пропорционально падающей мощности

$$
d \Phi=-\Phi_{0} d l / a .
$$

Распространение излучения носит диффузный характер. Поэтому любая взятая внутри среды по- верхность [4,5], например, перпендикулярная направлению $X$, видна под любым углом $\varphi$ с одинаковой яркостью $I$ :

$$
d \Phi_{\varphi}=I d \Omega,
$$

где $d \Omega$ - элемент телесного угла, в который попадает излучение. Для излучения, идущего под углом $\varphi$ к оси $X$,

$$
d \Omega=\sin \varphi d \varphi .
$$

Мощность, идущая через элемент площади $S$, перпендикулярный оси $X$,

$$
P=\int_{0}^{\pi / 2} S \cos \varphi d \Phi_{\varphi}=I S / 2 .
$$

В состоянии теплового равновесия в среде через любой элемент площади идет мощность, равная мощности, излучаемой с такого же элемента площади абсолютно черного тела. По закону Стефана-Больцмана мощность, излучаемая с элемента площадью $S$ поверхности абсолютно черного тела с температурой $T[4,5]$

$$
P=\sigma T^{4} S,
$$

где $\sigma$ - постоянная Стефана-Больцмана. Тогда

$$
I=2 \sigma T^{4}, \quad d \Phi_{\varphi}=2 \sigma T^{4} \sin \varphi d \varphi .
$$

Компонента плотности потока энергии, излучаемой вдоль оси $X$,

$$
\Phi_{x}=\int_{0}^{\pi / 2} \cos ^{2} \varphi d \Phi_{\varphi}=2 \sigma T^{4} / 3 .
$$

По закону Кирхгофа в состоянии теплового равновесия сколько мощности поглощается, столько же и излучается самой средой $[4,5]$, или, точнее, поглощаемая плотность потока энергии равна излучаемой. 


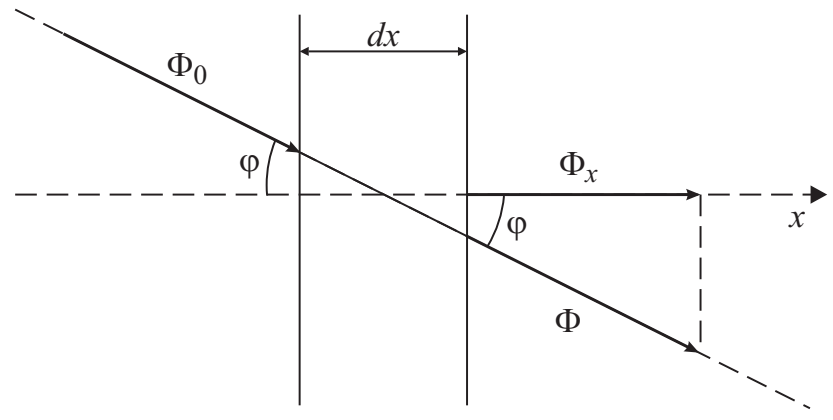

Рис. 1. Иллюстрация инвариантности светимости слоя.

Найдем компоненту плотности потока энергии (рис. 1), излучаемую нормально слою толщиной $d x$ при падении излучения на него под углом $\varphi$.

Поглощение (3) и соответственно излучение обратно пропорциональны косинусу угла падения

$$
d l=d x / \cos \varphi .
$$

$X$-компонента плотности потока энергии, наоборот, прямо пропорциональна косинусу угла падения. Изменение $X$-компоненты плотности потока одинаково независимо от направления падения. Поэтому светимость слоя нормально к поверхности не зависит от угла падения

$$
d \Phi_{x \varphi} / \Phi_{\varphi}=-d \Phi_{\varphi} \cos \varphi / \Phi_{\varphi}=d x / a .
$$

Для распространения энергии в заданном направлении не существен тот факт, что излучение идет диффузно. Плотность потока энергии, излучаемой нормально к поверхности полупрозрачного слоя толщиной $d x$,

$$
d \Phi_{x}=2 \sigma T^{4} d x /(3 a) .
$$

Для справедливости формулы (12) достаточно выполнения условия локального теплового равновесия, при котором температура слоя постоянна.

Пусть в среде вдоль оси $X$ будет некоторый небольшой градиент температуры $\alpha$ такой, что изменение температуры на толщине $a$ мало по сравнению с самой температурой

$$
\alpha=d T / d x \ll T / a .
$$

В этом случае поток энергии излучения с горячей стороны от поверхности не компенсируется потоком с холодной стороны (рис. 2). Видимость слоя в зависимости от расстояния $x$ до него, согласно (9), (12), убывает экспоненциально. Плотность потока энергии $d \Phi_{x}$ от элемента толщиной $d x$, находящегося на расстоянии $\mathrm{x}$ от поверхности с температурой $T$, составляет

$$
d \Phi_{x}=-2 \sigma(T+\alpha x)^{4} \exp (-x / a) d x /(3 a) .
$$

Некомпенсированная плотность потока энергии равна разности плотностей потока с холодной и горячей сто- рон от поверхности

$$
\begin{aligned}
\Phi_{\infty} & =\int_{0}^{\infty} d \Phi_{x}-d \Phi_{-x} \approx \frac{-16 \alpha \sigma T^{3}}{3 a} \int_{0}^{\infty} x \exp \left(\frac{-x}{a}\right) d x \\
& =\frac{-16 \alpha \sigma T^{3} a}{3}=-L \alpha .
\end{aligned}
$$

При обычных температурах, когда разность температур много меньше средней температуры, плотность потока энергии излучения, так же как и плотность потока, обусловленная кондуктивной теплопроводностью среды, пропорциональна градиенту температуры. Явление вязкого распространения излучения в серой среде называют диффузией теплового излучения $[4,5]$. Коэффициент пропорциональности между плотностью потока энергии излучения и градиентом температуры $L=16 \sigma T^{3} a / 3$ называют лучистой теплопроводностью среды [5]. Формула (15) как результат интегрирования верна только на расстояниях от границ среды, существенно превышающих глубину проникновения теплового излучения $a$ (в приближении большой оптической толщины среды). Заметим, что для формулы (14) это не так, она справедлива и возле диффузно излучающих (отражающих) границ среды. В условиях локального теплового равновесия при известной зависимости температуры от координаты и заданных граничных условиях формула (14) позволяет находить плотность потока энергии излучения в среде.

При постоянной приложенной разности температур поток энергии излучения, как и поток тепла при кондуктивной теплопроводности, обратно пропорционален толщине образца материала. Вследствие собственной тепловой светимости полупрозрачной среды изменение потока энергии излучения не сводится к экспоненциальному затуханию в толще образца. Долю мощности, переносимую излучением, часто без всяких на то оснований ошибочно включают в долю, переносимую благодаря кондуктивной теплопроводности. При измерениях выявляют зависимость, удовлетворяющую уравнению Фурье для теплопроводности, и полагают, что остальное несущественно. Но согласно (15), для излучения верна зависимость такого же типа. Наличие линейной зависимости плотности потока энергии от градиента

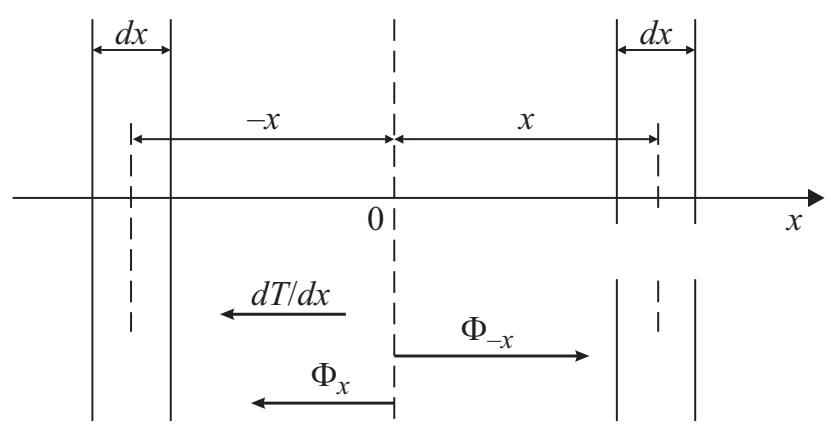

Рис. 2. Формирование плотности потока теплового излучения навстречу градиенту температуры в полупрозрачной среде. 
температуры еще не говорит о том, что вклад излучения в переносе тепла мал. Согласно уравнению Фурье, плотность потока энергии $\Phi_{h}$ благодаря кондуктивной теплопроводности среды $D$ :

$$
\Phi_{h}=-D \alpha
$$

Радиационная составляющая передаваемой мощности равна кондуктивной при эквивалентной глубине проникновения

$$
a_{e}=3 D /\left(16 \sigma T^{3}\right) \text {. }
$$

Параметры большинства теплозащитных материалов близки к теплопроводности воздуха $D_{B}=$ $=0.0262 \mathrm{~W} /(\mathrm{m} \cdot \mathrm{K})[6]$, которому соответствует $a_{e}=$ $=3.3 \mathrm{~mm}$. Уже по визуальным характеристикам многих теплозащитных материалов, по их прозрачности в видимом диапазоне света понятно, что их глубины проникновения теплового излучения, как минимум, сравнимы с этим значением. Это говорит о том, что доля потока излучения в тепловом переносе материалов, как минимум, существенна. А при высокой прозрачности среды (большой $a$ ) и малой кондуктивной теплопроводности $D$ вклад излучения может быть не только существенным, но даже и доминирующим. Кроме этого, надо отметить, что задача измерения вклада того и другого типа передаваемой мощности и нахождения собственных параметров среды является достаточно нетривиальной и сложной для решения.

Для уменьшения потока лучистой энергии через теплозащитные материалы применяют отражающие металлические экраны. Рассмотрим, какой будет плотность потока энергии излучения в полупрозрачной среде вблизи от плоской полностью отражающей поверхности при постоянном градиенте температуры, ей перпендикулярном. В отличие от случая бесконечной среды в некомпенсированный поток энергии нужно будет добавить излучение, отраженное от поверхности, и отнять шедшее из-за поверхности. На расстоянии $b$ от поверхности

$$
\begin{aligned}
& \Phi_{b} \approx \Phi_{\infty}+\frac{8 \alpha \sigma T^{3}}{3 a}\left(\int_{-b}^{\infty} \exp \left(\frac{-2 b-x}{a}\right) x d x\right. \\
& \left.-\int_{b}^{\infty} \exp \left(\frac{-b-x}{a}\right) x d x\right)=\Phi_{\infty}\left(1-\exp \left(\frac{-b}{a}\right)\right) .
\end{aligned}
$$

Сама по себе отражающая поверхность не светится, а плотность потока энергии при удалении от поверхности стремится к плотности потока в бесконечной среде. Поток энергии теплового излучения формируется на расстоянии порядка глубины проникновения $a$ от непрозрачной поверхности. При произвольном коэффициенте светимости поверхности $\varepsilon$

$$
\Phi_{b \varepsilon} \approx \Phi_{\infty}\left(1-\frac{2-\varepsilon}{2} \exp \left(\frac{-b}{a}\right)\right) .
$$

Плотность потока энергии при наличии поверхности в любом случае меньше плотности потока в бесконечной среде, так как в этом случае до заданного места не доходит излучение от элементов среды за поверхностью с большей температурой.

Исследование возмущения тепловой проницаемости полупрозрачной среды возле непрозрачных объектов и есть путь к решению задачи о разделении типов передаваемой мощности и нахождении параметров среды.

Формирование постоянного градиента температуры - задача сложная. На практике значительно проще задача формирования потока энергии с одинаковой плотностью. Будем полагать, что такой радиационнокондуктивный поток идет перпендикулярно поверхности с диффузной светимостью $\varepsilon$. На любом расстоянии от поверхности плотность потока складывается из радиационной $\Phi_{r}$ и кондуктивной $\Phi_{h}$ составляющих

$$
\Phi=\Phi_{h}+\Phi_{r}=\text { const. }
$$

На большом расстоянии от поверхности доли, приходящиеся на тот и другой тип, стремятся к константе:

$$
\Phi=\Phi_{h \infty}+\Phi_{r \infty}=-(D+L) \alpha_{\infty},
$$

и формируется постоянный градиент температуры $\alpha_{\infty}$. Вблизи от поверхности недостаток потока энергии излучения должен компенсироваться бо́льшим кондуктивным потоком и соответственно бо́льшим градиентом температуры, который в свою очередь изменяет поток энергии излучения. Детальный анализ показывает, что зависимость градиента температуры от расстояния от поверхности должна быть монотонной функцией, стремящейся к $\alpha_{\infty}$. Будем искать зависимость температуры от расстояния от поверхности в виде

$$
T=T_{0}+\alpha_{\infty} x+\beta \exp (-\gamma x / a)
$$

$\left(\beta \ll T_{0}\right)$, тогда плотность потока энергии излучения на расстоянии $b$ равна

$$
\begin{aligned}
& \Phi_{r b}=\frac{2 \sigma}{3}\left(\int_{0}^{b} T^{4} \exp \left(\frac{x-b}{a}\right) \frac{d x}{a}-\int_{b}^{\infty} T^{4} \exp \left(\frac{b-x}{a}\right) \frac{d x}{a}\right. \\
& \left.+\int_{0}^{\infty} T^{4}(1-\varepsilon) \exp \left(\frac{-x-b}{a}\right) \frac{d x}{a}+\varepsilon T_{0}^{4} \exp \left(\frac{-b}{a}\right)\right) \\
& \approx \Phi_{r \infty}\left(1-\frac{2-\varepsilon}{2} \exp \left(\frac{-b}{a}\right)+\frac{\beta}{a \alpha_{\infty}}\left(\frac{-\gamma}{1-\gamma^{2}} \exp \left(\frac{-\gamma b}{a}\right)\right.\right. \\
& \left.\left.+\frac{\gamma+\left(\gamma^{2}-\gamma\right) \varepsilon / 2}{1-\gamma^{2}} \exp \left(\frac{-b}{a}\right)\right)\right) .
\end{aligned}
$$

Плотность потока энергии благодаря кондуктивной теплопроводности на расстоянии $b$ равна

$$
\Phi_{h b}=-D\left(\alpha_{\infty}-\frac{\beta \gamma}{a} \exp \left(\frac{-\gamma b}{a}\right)\right) .
$$


Так как полная плотность потока энергии не должна зависеть от $b$, сумма коэффициентов при $\exp (-b / a)$ и $\exp (-\gamma b / a)$ должна быть равной 0 :

$$
-\frac{2-\varepsilon}{2}+\frac{\beta}{a \alpha_{\infty}} \frac{\gamma+\left(\gamma^{2}-\gamma\right) \varepsilon / 2}{1-\gamma^{2}}=0
$$

и

$$
\frac{-\gamma}{1-\gamma^{2}} \frac{\beta}{a \alpha_{\infty}} \Phi_{r \infty}+D \frac{\beta \gamma}{a}=0 .
$$

Из второго уравнения (25)

$$
\gamma^{2}=\Phi / \Phi_{h \infty}=(D+L) / D .
$$

Из первого уравнения (25)

$$
\beta=-a \alpha_{\infty} \frac{\gamma^{2}-1}{\gamma+\gamma^{2} \varepsilon /(2-\varepsilon)}
$$

Однозначность полученных коэффициентов $\beta$ и $\gamma$ подтверждает правильность выбранной зависимости (22).

Коэффициент $\gamma$ показывает, во сколько раз характерная толщина, на которой нелинейно изменяется температура возле непрозрачной поверхности, меньше глубины проникновения $a$ теплового излучения в среду. Из (26) $\gamma$ - заведомо больше 1. Чем выше доля мощности, идущей благодаря кондуктивной теплопроводности, тем $\gamma$ ближе к 1.

Коэффициент $\beta$ - это скачок температуры, возникающий вблизи непрозрачной поверхности. При малой доле мощности излучения скачок температуры тоже мал. При большой доле мощности излучения скачок температуры пропорционален $a$, а толщина, на которой он проявляется, пропорциональна корню из $а$. Скачок температуры пропорционален плотности потока идущей энергии. Его отношение к плотности потока энергии можно назвать поверхностным тепловым сопротивлением

$$
R=\frac{\beta}{\Phi}=\frac{a}{D+L} \frac{\gamma^{2}-1}{\gamma+\gamma^{2} \varepsilon /(2-\varepsilon)} .
$$

Градиент температуры в непосредственной близости от непрозрачной поверхности

$$
\alpha_{0+}=\alpha_{\infty}-\frac{\beta \gamma}{a}=\alpha_{\infty}\left(1+\frac{\gamma^{2}-1}{1+\gamma \varepsilon /(2-\varepsilon)}\right) .
$$

При $\varepsilon=0$

$$
\alpha_{0+}=\alpha_{\infty} \gamma^{2}=-\Phi / D
$$

То есть возле полностью отражающей поверхности, которая сама по себе не светится, поток тепла определяется только кондуктивной теплопроводностью среды.

Теперь, когда стало понятным поведение среды возле непрозрачной поверхности, рассмотрим еще более близкую к практике конструкцию из двух расположенных на расстоянии $d$ параллельных одинаковых поверхностей и полупрозрачной среды между ними. Между поверхностями приложена некоторая разность температур, много меньшая, чем их полусумма. Плотность потока энергии во всех точках среды направлена перпендикулярно поверхностям вдоль оси $X$ и одинакова. Градиент температуры направлен навстречу плотности потока энергии. В этом случае началом координат логично выбрать равноудаленную от поверхностей точку. Обозначим температуру в этой точке $T_{0}$. В силу симметрии отклонение температуры среды от среднего значения $T_{0}$ должно быть нечетной функцией координаты. С учетом (22) зависимость температуры среды от координаты должна иметь вид

$$
\begin{aligned}
T= & T_{0}+\alpha_{\infty} x+\psi(d)(\beta \exp (-\gamma(x+d / 2) / a) \\
& -\beta \exp (\gamma(x-d / 2) / a)),
\end{aligned}
$$

где $\psi$ - коэффициент, выражающий взаимное влияние поверхностей. С учетом (29), (30) производная этой зависимости в непосредственной близости от поверхности при $\pm d / 2$ должна быть равной $\alpha_{\infty}-\beta \gamma / a$,

$$
T_{x}^{\prime}\left( \pm \frac{d}{2}\right)=\alpha_{\infty}-\frac{\psi(d)(1+\exp (-\gamma d / a)) \beta \gamma}{a}=\alpha_{\infty}-\frac{\beta \gamma}{a}
$$

Тогда

$$
\psi(d)=(1+\exp (-\gamma d / a))^{-1} .
$$

Тот же коэффициент можно получить как сумму бесконечной геометрической прогрессии, возникающей при сложении потоков энергии излучения при многократном отражении между поверхностями

$$
\psi(d)=\sum_{i=0}^{\infty}(-1)^{i} \exp (-i \gamma d / a) .
$$

Из (31), (33) приложенная между поверхностями разность температур

$$
T\left(\frac{d}{2}\right)-T\left(\frac{-d}{2}\right)=\alpha_{\infty} d-2 \beta \operatorname{th}\left(\frac{\gamma d}{2 a}\right) .
$$

С помощью установки для бесконвекционных измерений тепловой проницаемости материалов [7] (рис. 3) были измерены зависимости мощности, идущей через образец, при заданной разности приложенных температур от толщины образца.

Чтобы направить всю выделяемую в нагревателе мощность через образец к холодильнику, вокруг него сделан тепловой экран, снабженный своим управляемым нагревателем. Температуру экрана, нагревателя $T_{h}$ и холодильника $T_{c}$ измеряем с помощью электронных цифровых датчиков и записываем в компьютер. Сигнал с датчика на экране используем для стабилизации его температуры. При измерениях нагреватель экрана автоматически включаем, когда его температура опускается ниже $39^{\circ} \mathrm{C}$, и выключаем, когда превышает $40^{\circ} \mathrm{C}$. Реально температура экрана изменялась в диапазоне от 38.5 до $41^{\circ} \mathrm{C}$ с периодом $30 \mathrm{~s}$. Так как время релаксации температуры системы экран-теплоизоляция много 


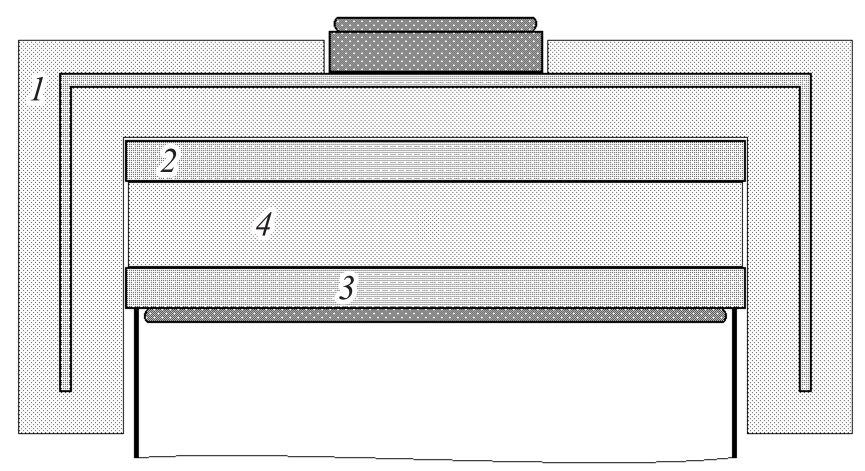

Рис. 3. Рабочая часть установки для измерения тепловой проницаемости материалов: 1 - медный тепловой экран внутри теплоизоляции из вспененного полистирола с управляемым нагревателем и радиатором, 2 - резистивный нагреватель, 3 - холодильник с радиатором отвода тепла, 4 - образец материала.

больше $30 \mathrm{~s}$, температуру экрана можно считать средней по периоду $\left(39.75^{\circ} \mathrm{C}\right)$. Температура холодильника благодаря подведенному водяному охлаждению оставалась примерно равной $20^{\circ} \mathrm{C}$.

При измерениях выделяемую в нагревателе электрическую мощность постепенно плавно увеличиваем, пока не достигнем стационарного состояния, в котором температура нагревателя не изменяется и равна температуре экрана. При этом поток тепла от нагревателя к экрану мал сразу по двум параметрам: и из-за малой теплопроводности слоя изоляции между ними и из-за малой разности температур между ними. Тогда можно считать, что вся выделяемая мощность $P=U^{2} / R_{h}$ идет через образец к холодильнику. Напряжение нагревателя $U$ измеряем с помощью вольтметра. Электрическое сопротивление нагревателя $R_{h}=20.0 \Omega$.

Вертикальная компоновка установки, при которой нагреватель находится над холодильником, гарантирует отсутствие конвективных потоков. При толщине образца меньшей, чем отношение площади поперечного сечения $72.3 \mathrm{~cm}^{2}$ к периметру $35.0 \mathrm{~cm}(<2 \mathrm{~cm})$, плотность потока энергии через образец можно считать в высокой степени параллельной, что соответствует проведенному выше теоретическому анализу.

В качестве резистивного нагревателя собрана плата из последовательно/параллельно соединенных чипрезисторов. Его внешней стороной является медненая поверхность платы. Рабочей поверхностью служат приклеенные эпоксидным клеем поверх резисторов (с заполнением всего свободного пространства внутри) пластины из монокристаллического кремния. Такая конструкция благодаря очень высокой теплопроводности меди и кремния обеспечивает высокую однородность температуры нагревателя. Энергетическая светимость внешней поверхности нагревателя мала, светимость рабочей поверхности, наоборот, высока, что позволяет учитывать мощность, идущую посредством теплового излучения. Рабочая поверхность холодильника тоже выполнена из монокристаллического кремния, пластины которого приклеены поверх керамических пластин из оксида алюминия, которые в свою очередь приклеены к медному радиатору водяного охлаждения. Она тоже обладает высокой энергетической светимостью.

Исследуемые образцы материала вырезаны строго по периметру рабочей поверхности установки из листа вспененного полиэтилена толщиной $d_{1}=2.2 \mathrm{~mm}$. Они имеют форму квадрата со стороной $85 \mathrm{~mm}$, площадью $S=72.3 \mathrm{~cm}^{2}$. Их масса $0.263 \mathrm{~g}$, плотность $16.5 \mathrm{~kg} / \mathrm{m}^{3}$, поверхностная плотность $36.4 \mathrm{~g} / \mathrm{m}^{2}$, доля заполненного полиэтиленом объема 0.0174 , размер пузырьков $0.2-0.5 \mathrm{~mm}$.

Для измерения зависимости от толщины образцы складываем в стопку с нужным числом слоев. Толщину стопки выставляем и контролируем с помощью четырех винтов, поддерживающих по углам шапку с экраном и нагревателем, надеваемую поверх столика с холодильником и образцами. В качестве экрана для излучения используем алюминиевую фольгу толщиной $10 \mu \mathrm{m}$. Толщина и тепловое сопротивление фольги пренебрежимо малы по сравнению с теми же параметрами образцов.

На рис. 4 представлены результаты измерений тепловой проницаемости стопок образцов с экранами и без экранов из фольги.

Согласно уравнению Фурье, зависимость отношения разности температур, приложенной к стопке образцов, к идущей через нее мощности от толщины стопки должна лечь на прямую линию. Это с хорошей точностью $(0.5 \%)$ получено для стопок образцов без фольги и для стопок образцов, с обеих сторон проложенных фоль-

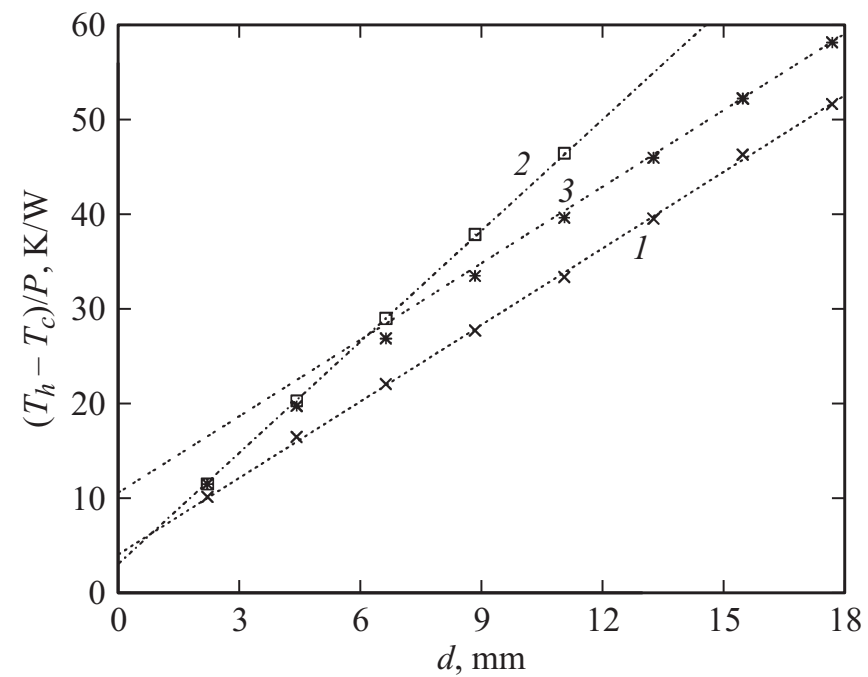

Рис. 4. Зависимости отношения разности температур к проходящей мощности от толщины стопки: 1 - для образцов без фольги и аппроксимирующая прямая, 2 - для всех образцов, с обеих сторон проложенных фольгой, и аппроксимирующая прямая, 3 - для стопки образцов, в которой фольга снизу от нижнего слоя (у холодильника) и сверху от верхнего (у нагревателя), и ее асимптота. 


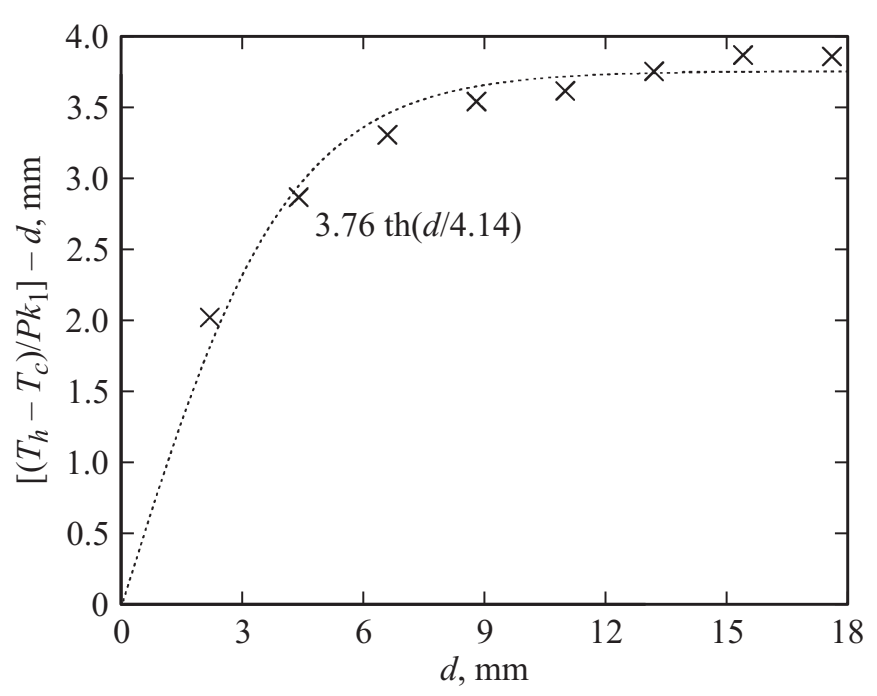

Рис. 5. Зависимость $\left(T_{h}-T_{c}\right) /\left(P k_{1}\right)-d$ от $d$ и аппроксимирующая кривая $A \operatorname{th}(d / B)$.

гой. Однако наклон зависимостей существенно отличен. В первом случае это $k_{1}=2.70 \mathrm{~K} /(\mathrm{W} \cdot \mathrm{mm})$ (свободный член $\left.y_{1}=4.0 \mathrm{~K} / \mathrm{W}\right)$, а во втором $k_{2}=3.92 \mathrm{~K} /(\mathrm{W} \cdot \mathrm{mm})$ $\left(y_{2}=3.0 \mathrm{~K} / \mathrm{W}\right)$. Для третьей зависимости, где проложены два экрана у нагревателя и у холодильника, видно, что при малых толщинах точки ложатся на зависимость, близкую ко второй прямой, а при больших толщинах асимптотически стремятся к прямой, параллельной первой.

Полученные результаты имеют простое и очевидное объяснение. Потоки радиационного и кондуктивного тепла примерно одинаковы. Глубина проникновения излучения больше, чем толщина одного образца (слоя). Поэтому стопки образцов без фольги пропускают оба вида потоков тепла, а для стопок образцов, проложенных фольгой, доля излучения менее существенна. Соответственно в первом случае наклон прямой $k_{1}=(S(D+L))^{-1}$ (см. (21)), а во втором случае $k_{2} \approx(S D)^{-1}$ (см. (30)). Наконец, для третьей зависимости при малых толщинах доля излучения мала. По мере увеличения толщины стопки, когда та становится сравнимой и превышает глубину проникновения излучения во вспененный полиэтилен, доля, обусловленная излучением, приближается к той же, что для образцов без фольги. Наличие фольги у нагревателя и у холодильника при этом проявляется в виде постоянной добавки, определяемой дополнительным тепловым сопротивлением на каждой отражающей поверхности (31), (33), (35).

Суммарная теплопроводность вспененного полиэтилена $D+L=\left(S k_{1}\right)^{-1}=(5.12 \pm 0.03) \cdot 10^{-2} \mathrm{~W} /(\mathrm{m} \cdot \mathrm{K})$, где $L=16 \sigma T_{0}^{3} a / 3-$ его коэффициент лучистой теплопроводности и $T_{0}=\left(T_{h}+T_{c}\right) / 2=304 \mathrm{~K}$.

Учитывая (21), $\alpha_{\infty}=P k_{1}$. Тогда из (27), (35) при $\varepsilon=0$

$$
\frac{T_{h}-T_{c}}{P k_{1}}-d=\frac{2 a\left(\gamma^{2}-1\right)}{\gamma} \operatorname{th}\left(\frac{\gamma d}{2 a}\right) \text {. }
$$

Производим с данными для третьей кривой (рис. 4) преобразования согласно выражению в левой части равенства (36) (делим на $k_{1}$ и вычитаем $d$ ) и затем аппроксимируем полученные данные зависимостью $A \operatorname{th}(d / B)$ (рис. 5).

Результаты численной аппроксимации $A=3.76 \pm 0.08 \mathrm{~mm}$ и $B=4.14 \pm 0.08 \mathrm{~mm}$. Тогда $A / B=$ $=\gamma^{2}-1=L / D=0.908 \quad$ (см. (26)), $\gamma=1.38 \pm 0.01$, $a=\gamma B / 2=2.86 \pm 0.06 \mathrm{~mm}$. Учитывая, что $D+L=$ $=5.12 \cdot 10^{-2} \mathrm{~W} /(\mathrm{m} \cdot \mathrm{K}), \quad$ получим коэффициент кондуктивной теплопроводности вспененного полиэтилена $D=(2.68 \pm 0.05) \cdot 10^{-2} \mathrm{~W} /(\mathrm{m} \cdot \mathrm{K})$ и его коэффициент лучистой теплопроводности $L=(2.44 \pm$ $\pm 0.05) \cdot 10^{-2} \mathrm{~W} /(\mathrm{m} \cdot \mathrm{K})$. Зная $L$, глубину проникновения теплового излучения во вспененный полиэтилен можно вычислить независимо по формуле (15). Расчетное значение $a^{\prime}=2.87 \pm 0.06 \mathrm{~mm}$ практически совпадает с $a$. Это подтверждает обоснованность проведенного выше теоретического анализа.

Толщина $a / \gamma=2.03 \mathrm{~mm}$, на которой формируется поток излучения в среде, действительно близка к толщине одного слоя вспененного полиэтилена. Однако при наличии образцов такого же материала, но меньшей толщины $(\sim 1 \mathrm{~mm})$ измерения могли бы быть в $2-3$ раза точнее. Тем не менее получено весьма разумное значение кондуктивной теплопроводности вспененного полиэтилена, чуть большее значения для воздуха. Доля лучистой составляющей в суммарной теплопроводности вспененного полиэтилена - чуть меньше половины.

Кроме измерений составляющих теплопроводности проведены непосредственные измерения дополнительного теплового сопротивления, которое возникает возле экрана в среде. Взяты стопки образцов, точно посередине которых помещен один-единственный экран из фольги (рис. 6).

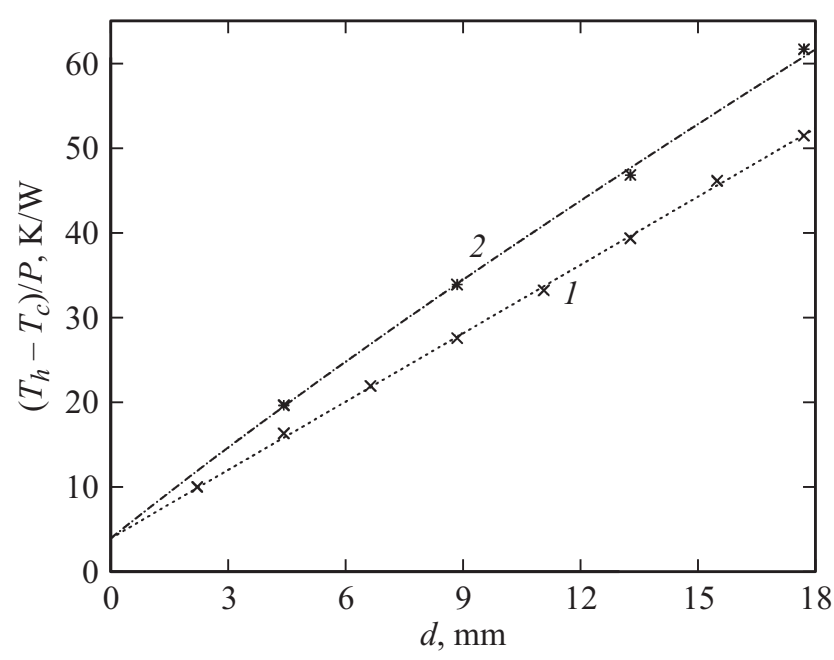

Рис. 6. Зависимости отношения разности температур, приложенной к стопке образцов, к идущей через нее мощности от толщины стопки: 1 - для образцов без фольги и аппроксимирующая прямая, 2 - для стопок образцов, в середине проложенных фольгой, и аппроксимирующая кривая. 
Данные для стопок с фольгой аппроксимированы зависимостью $\Delta T / P=k_{1} d+b_{1}+\delta\left(1-\exp \left(-d / d_{e}\right)\right)$ и найден коэффициент $\delta=10.0 \pm 0.5 \mathrm{~K} / \mathrm{W}$, пропорциональный тепловому сопротивлению $R$, возникающему у отражающей непрозрачной поверхности, $\delta=2 R / S$ (см. (28)). Тогда поверхностное тепловое сопротивление $R=(3.6 \pm 0.18) \cdot 10^{-2} \mathrm{~K} \cdot \mathrm{m}^{2} / \mathrm{W}$. То же тепловое сопротивление можно рассчитать по формуле (28), используя полученные ранее значения глубины проникновения $a=2.86 \mathrm{~mm}$, суммарной теплопроводности $D+L=0.0512 \mathrm{~W} /(\mathrm{m} \cdot \mathrm{K})$ и $\gamma=1.38$ при $\varepsilon=0$. Расчетное значение $R^{\prime}=3.67 \cdot 10^{-2} \mathrm{~K} \cdot \mathrm{m}^{2} / \mathrm{W}$ неплохо совпадает с измеренным, что опять-таки подтверждает правильность выполненного теоретического анализа.

Следует отметить, что воздух при столь малых толщинах, как в нашем эксперименте, можно считать совершенно прозрачным. Тепловое излучение реально поглощается только в самом полиэтилене. Известно [8], что полиэтиленовая пленка толщиной $800 \mu \mathrm{m}$ существенно поглощает излучение только в узких полосах вблизи длин волн 6.8 и $13.7 \mu \mathrm{m}$. В остальной части спектра теплового излучения пропускание пленки составляет около $60 \%$. Согласно нашим результатам, на глубине проникновения во вспененный полиэтилен $a=2.86 \mathrm{~mm}$ излучение проходит через слой полиэтилена толщиной $2.86 \cdot 0.0174=50 \mu \mathrm{m}$. Для поглощения в $(e-1) / e$ такой толщины, конечно, недостаточно. На самом деле глубина проникновения излучения в неоднородном по объему материале $a=(v+\chi)^{-1}$ определяется двумя параметрами: коэффициентом поглощения $\chi$ и коэффициентом рассеяния $v$ [4]. В нашем случае коэффициент рассеяния много больше коэффициента поглощения. Характерный размер элементов структуры материала много больше длины волны теплового излучения. Поэтому коэффициент рассеяния одинаков для всего спектра теплового излучения, и глубина проникновения $a$ от частоты излучения практически не зависит.

Благодаря большому рассеянию и сравнительно малому поглощению практически все мелкодисперсные легкие теплозащитные материалы можно с высокой точностью считать для теплового излучения серыми. Во всех таких материалах хорошо работает диффузное приближение для распространения теплового излучения. Но для не так хорошо рассеивающих материалов разработанный здесь подход может быть не столь эффективным.

Таким образом, проведен комплекс исследований теплового переноса в полупрозрачных материалах. На основании закона Кирхгофа (для теплового излучения) получены формулы для нахождения плотности потока энергии теплового излучения в среде при известной зависимости температуры от координаты. Определена зависимость температуры среды от расстояния до непрозрачного экрана при потоке энергии постоянной плотности при наличии и потока теплового излучения, и классической теплопроводности. Проведены измерения тепловой проницаемости. Разработаны методики определения показателей среды. На примере вспененного полиэтилена получены значения коэффициентов лучистой, кондуктивной и суммарной теплопроводности среды, глубины проникновения в нее теплового излучения и теплового сопротивления возле отражающего экрана в среде. Подтверждено и качественное, и количественное согласие теоретических оценок и экспериментальных результатов.

Вспененный полиэтилен - далеко не единственный материал, обладающий подобными свойствами. Например, измеренная суммарная теплопроводность известного утеплительного материала, выпускаемого под маркой „Холлофайбер“ (нетканого материала с объемной плотностью $13 \mathrm{~kg} / \mathrm{m}^{3}$ из полых волокон полиэтилентерефталата или лавсана толщиной $10 \mu \mathrm{m})$, составила $(4.77 \pm 0.04) \cdot 10^{-2} \mathrm{~W} /(\mathrm{m} \cdot \mathrm{K})$. С учетом результатов проведенных измерений с высокой вероятностью можно сказать, что доля излучения в тепловой проницаемости „Холлофайбера“ составляет около 40\%. „Холлофайбер“ пропускает тепловое излучение почти так же хорошо, как вспененный полиэтилен. Измеренная полная тепловая проводимость пенопласта (вспененного полистирола с плотностью $32 \mathrm{~kg} / \mathrm{m}^{3}$ и размером пузырьков $\sim 0.1 \mathrm{~mm}$ ) составила $(2.76 \pm 0.04) \cdot 10^{-2} \mathrm{~W} /(\mathrm{m} \cdot \mathrm{K})$. Доля излучения в его тепловой проводимости не превышает $10 \%$. Вспененный полистирол поглощает тепловое излучение, как минимум, в пять раз лучше, чем полиэтилен. Наиболее вероятно, что более высокое поглощение обусловлено существенно меньшим характерным размером элементов его структуры. При больших размерах пузырьков доля мощности, пропускаемая посредством излучения, должна быть существенно выше.

Работа выполнена при частичной поддержке Российского фонда фундаментальных исследований (грант 1602-00789).

\section{Список литературы}

[1] Тимофеев Ю.М., Шульгина Е.М. // Известия РАН. Физика атмосферы и океана. 2016. Т. 52, № 5. С. 529-545.

[2] Рубцов Н.А. // Теплофизика и аэромеханика. 2012. № 4. C. 521-531.

[3] Рубцов Н.А., Слепцов С.Д. // Теплофизика и аэромеханика. 2017. № 1. С. 109-113.

[4] Зигель Р., Хауэлл Джс. Теплообмен излучением / Пер. с англ. М.: Мир, 1975. 934 с.

[5] Кутателадзе С.С. Основы теории теплообмена. Изд. 5-е перераб. и доп. М.: Атомиздат, 1979. 416 с.

[6] Физические величины: Справочник / Под ред. И.С. Григорьева, Е.З. Мейлихова. М.: Энергоатомиздат, 1991. 1232 с.

[7] Полезная модель. №166709. Е.Ю. Шампаров, И.Н. Жагрина. Установка для прецизионных бесконвекционных измерений тепловой проницаемости материалов при температуpax, близких к комнатной. Зарегистрировано в Гос. реестре полезных моделей РФ 17 ноября 2016 г. www1.fips.ru.

[8] Кристаллические полиолефины: Сб. моногр. статей / Под ред. Р.А. Раффа, К.В. Дака. Т. 2. Строение и свойства. М.: Химия, 1970. 469 с. 